\title{
Generated Covariates in Nonparametric Estimation: A Short Review.
}

\author{
Enno Mammen* \\ Christoph Rothe** \\ Melanie Schienle***
}

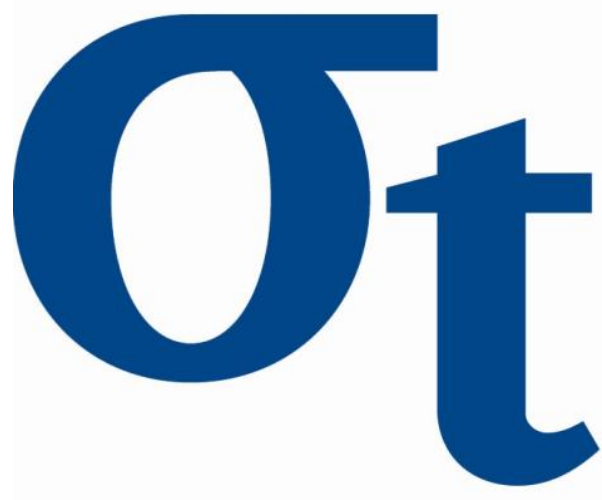

* Department of Economics, University of Mannheim,

** Toulouse School of Economics, France

*** Humboldt-Universität zu Berlin, Germany 


\title{
Generated Covariates in Nonparametric Estimation: A Short Review.
}

\author{
Enno Mammen, Christoph Rothe, and Melanie Schienle
}

\begin{abstract}
In many applications, covariates are not observed but have to be estimated from data. We outline some regression-type models where such a situation occurs and discuss estimation of the regression function in this context. We review theoretical results on how asymptotic properties of nonparametric estimators differ in the presence of generated covariates from the standard case where all covariates are observed. These results also extend to settings where the focus of interest is on average functionals of the regression function.
\end{abstract}

JEL Classification: C14, C31

Keywords: Nonparametric estimation, generated covariates

\section{Introduction}

Consider a nonparametric regression model of the form

$$
\begin{aligned}
& Y=m_{0}(R)+\varepsilon, \\
& E[\varepsilon \mid R]=0
\end{aligned}
$$

Enno Mammen

Department of Economics, University of Mannheim, D-68131 Mannheim, Germany, e-mail: emammen@rumms.uni-mannheim.de

Christoph Rothe

Toulouse School of Economics, 21 Alle de Brienne, F-31000 Toulouse, France e-mail: rothe@cict.fr

Melanie Schienle

School of Business and Economics, Humboldt University Berlin, Spandauer Str. 1, D-10178 Berlin, Germany e-mail: melanie.schienle@wiwi.hu-berlin.de. Research support from the DFG via the Collaborative Research Center 649 is gratefully acknowledged 
where $Y$ is a one-dimensional response variable and $R$ is a $q$-dimensional covariate vector. The statistical goal is to nonparametrically estimate the regression function $m_{0}: \mathbf{R}^{q} \rightarrow \mathbf{R}$ or a functional of the regression function, e.g. a weighted average $T\left(m_{0}\right)=\int m_{0}(x) w(x) d x$. We consider the case where the covariate $R$ is unobserved but an estimator $\widehat{R}$ of $R$ is available. In this note, we provide some examples where such a situation occurs. Furthermore, appropriate forms of nonparametric estimators of $m_{0}$ are discussed and results on their asymptotic distribution are reviewed. In particular, we analyse how the real feasible estimator of $m_{0}$ obtained via regression on $\widehat{R}$ differs from the infeasible one obtained by regressing on $R$. With stochastic expansions for the difference of these two estimators, the asymptotic distribution of the real estimator of $m_{0}$ can be accurately described.

The note is organized as follows. In the next section, some examples illustrate how and where generated covariates typically appear in practice. Section 3 provides an overview of the asymptotic theory when $m_{0}$ is estimated by local linear estimation. In particular, the theory can also be applied to cases where the main interest is in averages of the regression function $m_{0}$, which is also important for some of the stated examples.

\section{Examples}

\subsection{Simultaneous Nonparametric Equation Models without Additivity (Imbens and Newey, 2009)}

In economic models, there are often unobserved covariates which affect both response and observed covariates. Generally, such covariates which are correlated with the disturbance are called endogenous. Imbens and Newey (2009) propose a regression model with endogenous covariates where the error variable does not enter additively into the model. This allows for general forms of unobserved heterogeneity which has led to recent popularity of such nonseparable models among economists.

They consider a general regression relation of the form

$$
Y=\mu\left(X_{1}, Z_{1}, e\right)
$$

where $X_{1}$ and $Z_{1}$ are observed covariates and $Y$ is a one-dimensional response. While $Z_{1}$ is independent of the error variable $e$, no assumptions are made on the dependence between $X_{1}$ and $e$ at this stage. For identification, however, assume that the endogenous variable $X_{1}$ is generated as

$$
X_{1}=h\left(Z_{1}, Z_{2}, V\right),
$$

where $Z_{2}$ is an observed so-called instrumental variable not contained in the original equation, and $\left(Z_{1}, Z_{2}\right)$ is independent of the joint vector of errors $(e, V)$. 
If the function $h$ is strictly monotone in $V$, one can set without loss of generality that the conditional distribution of $V$ given $\left(Z_{1}, Z_{2}\right)$ is the uniform law on [0,1]. This can be achieved by putting

$$
V=F_{X_{1} \mid Z_{1}, Z_{2}}\left(X_{1}, Z_{1}, Z_{2}\right)
$$

and choosing $h$ as the inverse of $F_{X_{1} \mid Z_{1}, Z_{2}}$. Then by definition, the conditional distribution of $V$ given $\left(Z_{1}, Z_{2}\right)$ does not depend on $\left(Z_{1}, Z_{2}\right)$. Thus, $V$ is independent of $\left(Z_{1}, Z_{2}\right)$. Note that the above independence assumption is slightly more restrictive, because it does not only require that $\left(Z_{1}, Z_{2}\right)$ is independent of each $e$ and $V$ separately, but also of $(e, V)$ jointly.

For fixed values of $z_{1}, z_{2}$ and $v$ and for $x_{1}=h\left(z_{1}, z_{2}, v\right)$ it is straightforward to show

$$
\begin{aligned}
& E\left[\mu\left(x_{1}, z_{1}, e\right) \mid V=v\right] \\
& =E\left[\mu\left(X_{1}, Z_{1}, e\right) \mid Z_{1}=z_{1}, Z_{2}=z_{2}, V=v\right] \\
& =E\left[\mu\left(X_{1}, Z_{1}, e\right) \mid X_{1}=x_{1}, Z_{1}=z_{1}, V=v\right] \\
& =E\left[Y \mid Z_{1}=z_{1}, Z_{2}=z_{2}, V=v\right] .
\end{aligned}
$$

Thus we can write

$$
Y=m_{0}(R)+\varepsilon
$$

where

$$
\begin{aligned}
& S=\left(X_{1}, Z_{1}, Z_{2}\right), \\
& R=r_{0}(S)=\left(X_{1}, Z_{1}, F_{X_{1} \mid Z_{1}, Z_{2}}\left(X_{1}, Z_{1}, Z_{2}\right)\right)=\left(X_{1}, Z_{1}, V\right), \\
& m_{0}\left(x_{1}, z_{1}, v\right)=E\left[\mu\left(x_{1}, z_{1}, e\right) \mid V=v\right], \\
& \varepsilon=Y-E[Y \mid S] .
\end{aligned}
$$

In this model, the covariate $V$ is unobserved, but an estimate

$$
\hat{V}=\hat{F}_{X_{1} \mid Z_{1}, Z_{2}}\left(X_{1}, Z_{1}, Z_{2}\right)
$$

of $V$ is available. Thus, instead of $R$ also use the feasible $\hat{R}=\left(X_{1}, Z_{1}, \hat{V}\right)$. Then the function $m_{0}$ can be estimated by regressing $Y$ onto $\hat{R}$. Let us denote this estimator as real, feasible estimator $\hat{m}$. One may compare this estimator to the theoretical, infeasible estimator $\tilde{m}$ obtained from regressing $Y$ onto $R$. If the asymptotics of the theoretical estimator $\tilde{m}$ are well-understood, an asymptotic understanding of $\hat{m}$ can be based on a stochastic expansion of the difference of $\hat{m}-\tilde{m}$.

The function $m_{0}$ is not of direct interest because it contains the nuisance covariate $V$. In general, the focus is on the so-called average structural function $E\left[\mu\left(x_{1}, z_{1}, e\right)\right]$, the expected response if one exogenously fixes $X_{1}$ at $x_{1}$ and $Z_{1}$ at $z_{1}$. This function can be estimated by

$$
\int_{0}^{1} \widehat{m}\left(x_{1}, z_{1}, v\right) d v
$$


Other functionals of interest are averages of the derivative $\partial \mu\left(x_{1}, z_{1}, e\right) / \partial\left(x_{1}, z_{1}\right)$.

\subsection{Simultaneous Nonparametric Equation Models with Additivity (Newey, Powell, Vella 1999)}

In Newey, Powell, Vella (1999) a submodel of the regression model of the last subsection is considered. The setup differs from the last subsection by assuming that the error enters additively into the regression function, i.e.

$$
Y=\mu\left(X_{1}, Z_{1}\right)+e .
$$

For the control equation also an additive specification is used:

$$
X_{1}=h\left(Z_{1}, Z_{2}\right)+V,
$$

but one could also proceed with the control equation of the last section.

With $\left(Z_{1}, Z_{2}\right)$ independent of $(e, V)$ as before, it is

$$
E\left[Y \mid X_{1}, Z_{1}, Z_{2}\right]=\mu\left(X_{1}, Z_{1}\right)+\lambda(V)=E\left[Y \mid X_{1}, Z_{1}, V\right]
$$

with $\lambda(V)=E[e \mid V]$. Thus we get an additive model where the regressor in the second additive component is not observed. This additive model can also be obtained under slightly weaker conditions, namely that $E\left[e \mid Z_{1}, Z_{2}, V\right]=E[e \mid V]$ and $E\left[V \mid Z_{1}, Z_{2}\right]=0$.

There are two major approaches to fit an additive nonparametric model: marginal integration and backfitting. In Marginal Integration (Newey (1994), Tjøstheim and Auestad (1994), Linton and Nielsen (1995)), first a full dimensional regression function $E\left[Y \mid X_{1}=x_{1}, Z_{1}=z_{1}, V=v\right]$ is estimated. And then in a second step, $v$ is integrated out to obtain an estimate of $\mu\left(x_{1}, z_{1}\right)$. The first step of this procedure can be rewritten as a regression problem $Y=m_{0}(R)+\varepsilon$ with unobserved regressor $R$ where

$$
\begin{aligned}
& S=\left(X_{1}, Z_{1}, Z_{2}\right), \\
& R=r_{0}(S)=\left(X_{1}, Z_{1}, X_{1}-h\left(Z_{1}, Z_{2}\right)\right)=\left(X_{1}, Z_{1}, V\right), \\
& m_{0}(r)=E[Y \mid R=r], \\
& \varepsilon=Y-E[Y \mid R] .
\end{aligned}
$$

A fit of the unobserved $R$ is given by $\hat{R}=\left(X_{1}, Z_{1}, \hat{V}\right)$ with $\hat{V}=X_{1}-\hat{h}\left(Z_{1}, Z_{2}\right)$ where $\hat{h}$ is a (nonparametric) estimator of the control function $h$.

In the Smooth Backfitting approach (Mammen, Linton, Nielsen, 1999) for an additive model, estimates are obtained by iteration. As ingredients for the iteration algorithm, one needs estimators of the marginal expectations $E\left[Y \mid X_{1}, Z_{1}\right], E[Y \mid V]$, and of the joint density of $\left(X_{1}, Z_{1}, V\right)$. Here estimation of $E[Y \mid V]$ can be rewritten as 
a regression problem $Y=m_{0}(R)+\varepsilon$ with unobserved regressor $R$ where now

$$
\begin{aligned}
& S=\left(X_{1}, Z_{1}, Z_{2}\right), \\
& R=r_{0}(S)=X_{1}-h\left(Z_{1}, Z_{2}\right)=V, \\
& m_{0}(v)=E[Y \mid V=v], \\
& \varepsilon=Y-E[Y \mid V] .
\end{aligned}
$$

\subsection{Marginal Treatment Effects (Heckman, Vytlacil, 2005, 2009)}

In Heckman, Vytlacil $(2005,2009)$ the following model for treatment effects is discussed: we observe $D, Y_{D}, X, Z$ in

$$
\begin{aligned}
& Y_{d}=\rho\left(X, U_{d}, \theta_{d}\right) \quad \text { for } d=0,1 \\
& D=1, \text { if } V \leq \mu(Z), \text { and } D=0, \text { otherwise. }
\end{aligned}
$$

Here $\theta_{0}$ and $\theta_{1}$ are unknown parameters that are finite or infinite-dimensional. Furthermore, $\rho$ is a known function. An example for a specification would be $\rho\left(X, U_{d}, \theta_{d}\right)=m_{d}(X)+U_{d}$ with a "nonparametric parameter" $\theta_{d}=m_{d}$. The variable $D$ is a dummy variable that indicates if a person is treated or not. The model contains counterfactual outcomes. If a person is treated $(D=1)$ the outcome $Y_{1}$ is observed, assuming that there also exists an unobserved outcome $Y_{0}$ that would have been observed if the person had not been treated. The participation of the person in the treatment is driven by an unobserved variable $V$. Without loss of generality, set $V$ as uniform distribution on $[0,1]$. For identification of the model the following condition is required:

$\left(U_{0}, V\right)$ and $\left(U_{1}, V\right)$ are conditionally independent of $Z$ given $X$.

Note that the norming of $V$ implies that $P(D=1 \mid Z)=\mu(Z)$.

Here, a function of interest is the Marginal Treatment Effect $\operatorname{MTE}(x, v)=$ $E\left[Y_{1}-Y_{0} \mid X=x, V=v\right]$, the expected treatment effect for an individual with covariate $X=x$ that lies on the $v$-quantile of the unobserved propensity to participate in the treatment. It holds that

$$
\begin{aligned}
\operatorname{MTE}(x, v) & =E\left[Y_{1}-Y_{0} \mid X=x, V=v\right] \\
& =-\frac{\partial}{\partial v} E\left[Y_{D} \mid X=x, \mu(Z)=v\right] .
\end{aligned}
$$

This follows because for $\delta>0$ small:

$$
\begin{aligned}
& \operatorname{MTE}(x, v)=E\left[Y_{1}-Y_{0} \mid X=x, V=v\right] \\
& \begin{array}{l}
\approx \\
\delta^{-1}\left(-E\left[Y_{1} I[V \geq v+\delta] \mid X=x\right]-E\left[Y_{0} I[V<v+\delta] \mid X=x\right]\right. \\
\left.\quad+E\left[Y_{1} I[V \geq v] \mid X=x\right]+E\left[Y_{0} I[V<v] \mid X=x\right]\right) \\
=\delta^{-1}\left(-E\left[Y_{1} I[V \geq v+\delta] \mid X=x, \mu(Z)=v+\delta\right]\right.
\end{array}
\end{aligned}
$$




$$
\begin{aligned}
&-E\left[Y_{0} I[V<v+\delta] \mid X=x, \mu(Z)=v+\delta\right]+E\left[Y_{1} I[V \geq v] \mid X=x, \mu(Z)=v\right] \\
&\left.+E\left[Y_{0} I[V<v] \mid X=x, \mu(Z)=v\right]\right) \\
&=\delta^{-1}\left(-E\left[Y_{D} I[V \geq v+\delta] \mid X=x, \mu(Z)=v+\delta\right]\right. \quad-E\left[Y_{D} I[V<v+\delta] \mid X=x, \mu(Z)=v+\delta\right]+E\left[Y_{D} I[V \geq v] \mid X=x, \mu(Z)=v\right] \\
&\left.\quad+E\left[Y_{D} I[V<v] \mid X=x, \mu(Z)=v\right]\right) \\
&=\delta^{-1}\left(-E\left[Y_{D} \mid X=x, \mu(Z)=v+\delta\right]+E\left[Y_{D} \mid X=x, \mu(Z)=v\right]\right) \\
& \approx- \frac{\partial}{\partial v} E\left[Y_{D} \mid X=x, \mu(Z)=v\right] .
\end{aligned}
$$

Here estimation of (the partial derivative of) $E\left[Y_{D} \mid X=x, \mu(Z)=v\right]$ can be rewritten as a regression problem $Y=m_{0}(R)+\varepsilon$ with unobserved regressor $R$ where now

$$
\begin{aligned}
& Y=Y_{D}, \\
& S=(X, Z), \\
& R=r_{0}(S)=(X, \mu(Z)), \\
& m_{0}(r)=E[Y \mid(X, \mu(Z))=r], \\
& \varepsilon=Y_{D}-E\left[Y_{D} \mid(X, \mu(Z))\right] .
\end{aligned}
$$

Many treatment effects parameters and other parameters can be written as weighted averages of $\operatorname{MTE}(x, v)$. Estimation of the $M T E$ function is again based on a regression problem with an unobserved covariate $\mu(Z)$. Here interest is in a partial derivative of the regression function.

\subsection{Further Examples.}

Further examples of regression problems with unobserved covariates are sample selection models, censored regression models, generalized Roy models, stochastic volatility models and semiparametric GARCH-in-Mean models. For a discussion and/or references of these models we refer to Mammen, Rothe and Schienle (2011).

\section{Nonparametric Regression with Nonparametrically Generated Covariates.}

In all examples of the last section, the fit $\hat{R}$ of the unobserved covariate is of the form $\hat{R}=\hat{r}(S)$, where $\hat{r}$ is an estimator of a function $r_{0}$ that fulfills $R=r_{0}(S)$ for an observed covariate $S$. Thus we have the following nonparametric regression model

$$
\begin{aligned}
& Y=m_{0}\left(r_{0}(S)\right)+\varepsilon, \\
& E[\varepsilon \mid S]=0 .
\end{aligned}
$$


In this section, we give a brief description of the asymptotics of a nonparametric estimator $\hat{m}$ that is based on regressing $Y$ onto the fitted covariate $\hat{R}=\hat{r}(S)$. For illustration, we restrict the discussion to the special case where $\hat{m}=\widehat{m}_{L L}$ is a local linear estimator for an i.i.d. sample $\left(S_{i}, Y_{i}\right)$, i.e. $\widehat{m}_{L L}(x)=\widehat{\alpha}$, where $(\widehat{\alpha}, \widehat{\beta})$ minimizes

$$
\sum_{i=1}^{n}\left[Y_{i}-\alpha-\beta^{T}\left(\widehat{R}_{i}-x\right)\right]^{2} K_{h}\left(\widehat{R}_{i}-x\right)
$$

Here is $K_{h}(u)$ a product kernel:

$$
K_{h}(u)=\left(h_{1} \cdot \ldots \cdot h_{q}\right)^{-1} K_{1}\left(u_{1}\right) \cdot \ldots \cdot K_{q}\left(u_{q}\right)
$$

for kernel functions $K_{1}, \ldots, K_{q}$ and a bandwidth vector $h=\left(h_{1}, \ldots, h_{q}\right)$. We call this estimator also the real estimator, in contrast to the theoretical estimator $\widetilde{m}_{L L}$ which is defined as $\widetilde{m}_{L L}(x)=\widetilde{\alpha}$ where $(\widetilde{\alpha}, \widetilde{\beta})$ minimizes

$$
\sum_{i=1}^{n}\left[Y_{i}-\alpha-\beta^{T}\left(R_{i}-x\right)\right]^{2} K_{h}\left(R_{i}-x\right) .
$$

Since the $R_{i}$ 's are unobserved, this theoretical estimator is infeasible. It is, however, introduced here because its asymptotic behaviour is well understood. Thus, for the asymptotic properties of the real estimator we only need a stochastic expansion of $\widehat{m}_{L L}(x)-\widetilde{m}_{L L}(x)$. Such an expansion was derived in Mammen, Rothe and Schienle [6] (MRS in the following). Tailored results for parameters obtained as functionals of $m$ are derived in Mammen, Rothe and Schienle [7].

For the comparison of $\widehat{m}_{L L}$ and $\widetilde{m}_{L L}$, MRS use three types of assumptions: besides standard smoothing assumptions, these are conditions on accuracy (A) and complexity (C) of the estimator $\hat{r}$ of $r_{0}$. The assumption (A) requires that $\hat{r}$ converges to $r_{0}$ with a rate that is fast enough. The assumption (C) states that there exist sequences of sets $\mathscr{M}_{n}$ with two properties: (i) $\widehat{r} \in \mathscr{M}_{n}$ with probability tending to one. (ii) The sets $\mathscr{M}_{n}$ are not too large, where size is measured by entropy. The main result in MRS is the following expansion

$$
\widehat{m}_{L L}(x)-\widetilde{m}_{L L}(x) \approx-m^{\prime}(x) \frac{\frac{1}{n} \sum_{i=1}^{n} K_{h}\left(r_{0}\left(S_{i}\right)-x\right)\left(\widehat{r}\left(S_{i}\right)-r_{0}\left(S_{i}\right)\right)}{\frac{1}{n} \sum_{i=1}^{n} K_{h}\left(r_{0}\left(S_{i}\right)-x\right)} .
$$

This result can be interpreted as follows: The real estimator $\widehat{m}_{L L}(x)$ and the oracle estimator $\widetilde{m}_{L L}(x)$ differ by a local weighted average of the estimator of $r_{0}$ :

$$
-m^{\prime}(x) \frac{\frac{1}{n} \sum_{i=1}^{n} K_{h}\left(r_{0}\left(S_{i}\right)-x\right)\left(\widehat{r}\left(S_{i}\right)-r_{0}\left(S_{i}\right)\right)}{\frac{1}{n} \sum_{i=1}^{n} K_{h}\left(r_{0}\left(S_{i}\right)-x\right)} .
$$

This local average is of the order of the bias of $\widehat{r}$ but it may have a faster rate as the variance part of $\widehat{r}$. Thus we can conclude that for achieving a certain rate of convergence for estimating $m_{0}$, it is not necessary that an estimator of $r_{0}$ has the 
same or a faster rate. A similar result can be obtained for derivatives of the regression function.

We now shortly outline the main ideas of how the expansion of $\widehat{m}_{L L}(x)-\widetilde{m}_{L L}(x)$ was obtained in MRS. We want to compare:

real estimator $\widehat{m}_{L L}=$ SMOOTH of $\widehat{r}(S)$ versus $m_{0}\left(r_{0}(S)\right)+\varepsilon$,

oracle estimator $\widetilde{m}_{L L}=\mathrm{SMOOTH}$ of $r_{0}(S)$ versus $m_{0}\left(r_{0}(S)\right)+\varepsilon$.

Now, because of additivity of the operator SMOOTH, it is

$$
\begin{aligned}
\widehat{m}_{L L}=\text { SMOOTH of } \widehat{r}(S) \text { versus } m_{0}(\widehat{r}(S))+\varepsilon & \\
& + \text { SMOOTH of } \widehat{r}(S) \text { versus } m_{0}\left(r_{0}(S)\right)-m_{0}(\widehat{r}(S)) .
\end{aligned}
$$

If $\widehat{r}$ was non-random we get, because $\left|\widehat{r}(S)-r_{0}(S)\right|$ is small by assumption (A),

$$
\begin{aligned}
\widehat{m}_{L L} \approx & \text { SMOOTH of } r_{0}(S) \text { versus } m_{0}\left(r_{0}(S)\right)+\varepsilon \\
& \quad+\text { SMOOTH of } \widehat{r}(S) \text { versus } m_{0}\left(r_{0}(S)\right)-m_{0}(\widehat{r}(S)) \\
\approx \widetilde{m}_{L L} & \quad \text { SMOOTH of } r_{0}(S) \text { versus } m_{0}^{\prime}\left(r_{0}(S)\right)\left(r_{0}(S)-\widehat{r}(S)\right) .
\end{aligned}
$$

This is (nearly) the formula of the desired expansion.

It remains to take care of the fact that $\widehat{r}$ is random and not purely deterministic. In order to do so, the argument must be uniform over the set of possible realizations of $\widehat{r}$. This can be achieved by an empirical process worst case analysis. We must show that

$$
\begin{aligned}
\mid \widehat{m}_{L L, r}(x) & -\widetilde{m}_{L L}(x) \\
& +m^{\prime}(x) \frac{\frac{1}{n} \sum_{i=1}^{n} K_{h}\left(r_{0}\left(S_{i}\right)-x\right)\left(r\left(S_{i}\right)-r_{0}\left(S_{i}\right)\right)}{\frac{1}{n} \sum_{i=1}^{n} K_{h}\left(r_{0}\left(S_{i}\right)-x\right)} \mid
\end{aligned}
$$

is small uniformly for $r$ in $\mathscr{M}_{n}$. Here $\widehat{m}_{L L, r}$ is the local linear estimator based on regressing $Y$ onto $r(S)$. At this stage of the proof one makes use of Assumption (C).

\section{References}

1. Heckman, J.J. and Vytlacil, E. J.: Structural equations, treatment effects, and econometric policy evaluation. Econometrica 73, 669-738 (2005)

2. Heckman, J.J. and Vytlacil, E. J.: Econometric Evaluation of Social Programs, Part II: Using the Marginal Treatment Effect to Organize Alternative Econometric Estimators to Evaluate Social Programs, and to Forecast their Effects in New Environments. In: Heckman, J.J. and Leamer, E.E. (eds.) Handbook of Econometrics 6, chapter 71, Elsevier (2007)

3. Imbens, G.W. and Newey, W.K.: Identification and Estimation of Triangular Simultaneous Equations Models Without Additivity. Econometrica 77, 1481-1512 (2009)

4. Linton, O. and Nielsen, J.P.: Kernel estimation of partial means and a general variance estimator. Biometrika 82, 93-100 (1995) 
5. Mammen, E. , Linton, O. and Nielsen, J.P.: The existence and asymptotic properties of a backfitting algorithm under weak conditions. Annals of Statistics 27, 1443-1490 (1999)

6. Mammen, E. , Rothe, C. and Schienle, M. : Nonparametric regression with nonparametrically generated covariates. The Annals of Statistics, forthcoming (2012)

7. Mammen, E., Rothe, C. and Schienle, M.: Semiparametric estimation with generated covariates. Preprint (2012)

8. Newey, W.K.: A kernel method of estimating structured nonparametric regression based on marginal integration. Econometric Theory 10, 233-253 (1994)

9. Newey, W.K. and Powell, J.L. and Vella, F.: Nonparametric estimation of triangular simultaneous equations models. Econometrica 67, 565-603 (1999)

10. Tjøstheim, D. and Auestad, B.H.: Nonparametric Identification of Nonlinear Time Series: Selecting Significant Lags. Journal of the American Statistical Association, 89, (1994) 


\section{SFB 649 Discussion Paper Series 2012}

For a complete list of Discussion Papers published by the SFB 649, please visit http://sfb649.wiwi.hu-berlin.de.

001 "HMM in dynamic HAC models" by Wolfgang Karl Härdle, Ostap Okhrin and Weining Wang, January 2012.

002 "Dynamic Activity Analysis Model Based Win-Win Development Forecasting Under the Environmental Regulation in China" by Shiyi Chen and Wolfgang Karl Härdle, January 2012.

003 "A Donsker Theorem for Lévy Measures" by Richard Nickl and Markus Reiß, January 2012.

004 "Computational Statistics (Journal)" by Wolfgang Karl Härdle, Yuichi Mori and Jürgen Symanzik, January 2012.

005 "Implementing quotas in university admissions: An experimental analysis" by Sebastian Braun, Nadja Dwenger, Dorothea Kübler and Alexander Westkamp, January 2012.

006 "Quantile Regression in Risk Calibration" by Shih-Kang Chao, Wolfgang Karl Härdle and Weining Wang, January 2012.

007 "Total Work and Gender: Facts and Possible Explanations" by Michael Burda, Daniel S. Hamermesh and Philippe Weil, February 2012.

008 "Does Basel II Pillar 3 Risk Exposure Data help to Identify Risky Banks?" by Ralf Sabiwalsky, February 2012.

009 "Comparability Effects of Mandatory IFRS Adoption" by Stefano Cascino and Joachim Gassen, February 2012.

010 "Fair Value Reclassifications of Financial Assets during the Financial Crisis" by Jannis Bischof, Ulf Brüggemann and Holger Daske, February 2012.

011 "Intended and unintended consequences of mandatory IFRS adoption: A review of extant evidence and suggestions for future research" by Ulf Brüggemann, Jörg-Markus Hitz and Thorsten Sellhorn, February 2012.

012 "Confidence sets in nonparametric calibration of exponential Lévy models" by Jakob Söhl, February 2012.

013 "The Polarization of Employment in German Local Labor Markets" by Charlotte Senftleben and Hanna Wielandt, February 2012.

014 "On the Dark Side of the Market: Identifying and Analyzing Hidden Order Placements" by Nikolaus Hautsch and Ruihong Huang, February 2012.

015 "Existence and Uniqueness of Perturbation Solutions to DSGE Models" by Hong Lan and Alexander Meyer-Gohde, February 2012.

016 "Nonparametric adaptive estimation of linear functionals for low frequency observed Lévy processes" by Johanna Kappus, February 2012.

017 "Option calibration of exponential Lévy models: Implementation and empirical results" by Jakob Söhl und Mathias Trabs, February 2012.

018 "Managerial Overconfidence and Corporate Risk Management" by Tim R. Adam, Chitru S. Fernando and Evgenia Golubeva, February 2012.

019 "Why Do Firms Engage in Selective Hedging?" by Tim R. Adam, Chitru S. Fernando and Jesus M. Salas, February 2012.

020 "A Slab in the Face: Building Quality and Neighborhood Effects" by Rainer Schulz and Martin Wersing, February 2012.

021 "A Strategy Perspective on the Performance Relevance of the CFO" by Andreas Venus and Andreas Engelen, February 2012.

022 "Assessing the Anchoring of Inflation Expectations" by Till Strohsal and Lars Winkelmann, February 2012.

\section{SFB 649, Spandauer Straße 1, D-10178 Berlin http: //sfb649.wiwi.hu-berlin.de}

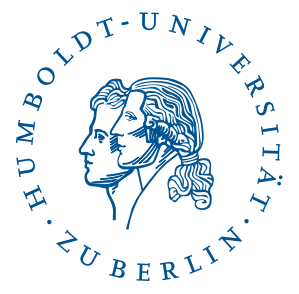




\section{SFB 649 Discussion Paper Series 2012}

For a complete list of Discussion Papers published by the SFB 649, please visit http://sfb649.wiwi.hu-berlin.de.

023 "Hidden Liquidity: Determinants and Impact" by Gökhan Cebiroglu and Ulrich Horst, March 2012.

024 "Bye Bye, G.I. - The Impact of the U.S. Military Drawdown on Local German Labor Markets" by Jan Peter aus dem Moore and Alexandra Spitz-Oener, March 2012.

025 "Is socially responsible investing just screening? Evidence from mutual funds" by Markus Hirschberger, Ralph E. Steuer, Sebastian Utz and Maximilian Wimmer, March 2012.

026 "Explaining regional unemployment differences in Germany: a spatial panel data analysis" by Franziska Lottmann, March 2012.

027 "Forecast based Pricing of Weather Derivatives" by Wolfgang Karl Härdle, Brenda López-Cabrera and Matthias Ritter, March 2012.

028 "Does umbrella branding really work? Investigating cross-category brand loyalty" by Nadja Silberhorn and Lutz Hildebrandt, April 2012.

029 "Statistical Modelling of Temperature Risk" by Zografia Anastasiadou, and Brenda López-Cabrera, April 2012.

030 "Support Vector Machines with Evolutionary Feature Selection for Default Prediction" by Wolfgang Karl Härdle, Dedy Dwi Prastyo and Christian Hafner, April 2012.

031 "Local Adaptive Multiplicative Error Models for High-Frequency Forecasts" by Wolfgang Karl Härdle, Nikolaus Hautsch and Andrija Mihoci, April 2012.

032 "Copula Dynamics in CDOs." by Barbara Choroś-Tomczyk, Wolfgang Karl Härdle and Ludger Overbeck, May 2012.

033 "Simultaneous Statistical Inference in Dynamic Factor Models" by Thorsten Dickhaus, May 2012.

034 "Realized Copula" by Matthias R. Fengler and Ostap Okhrin, Mai 2012.

035 "Correlated Trades and Herd Behavior in the Stock Market" by Simon Jurkatis, Stephanie Kremer and Dieter Nautz, May 2012

036 "Hierarchical Archimedean Copulae: The HAC Package" by Ostap Okhrin and Alexander Ristig, May 2012.

037 "Do Japanese Stock Prices Reflect Macro Fundamentals?" by Wenjuan Chen and Anton Velinov, May 2012.

038 "The Aging Investor: Insights from Neuroeconomics" by Peter N. C. Mohr and Hauke R. Heekeren, May 2012.

039 "Volatility of price indices for heterogeneous goods" by Fabian Y.R.P. Bocart and Christian M. Hafner, May 2012.

040 "Location, location, location: Extracting location value from house prices" by Jens Kolbe, Rainer Schulz, Martin Wersing and Axel Werwatz, May 2012.

041 "Multiple point hypothesis test problems and effective numbers of tests" by Thorsten Dickhaus and Jens Stange, June 2012

042 "Generated Covariates in Nonparametric Estimation: A Short Review." by Enno Mammen, Christoph Rothe, and Melanie Schienle, June 2012.

\section{SFB 649, Spandauer Straße 1, D-10178 Berlin} http://sfb649.wiwi.hu-berlin.de 